\title{
Measuring Threshold Potentials of Neuron Cells Using Hodgkin-Huxley Model by Applying Different Types of Input Signals
}

\author{
Molla Manjurul Islam and Naimul Islam* \\ *Department of Physics, Dhaka University, Dhaka-1000, Bangladesh.
}

(Received: 20 April 2015; Accepted: 15 November 2015)

\begin{abstract}
The Hodgkin-Huxley model is the first successful mathematical model for explaining the initiation and propagation of an action potential in a neuron cell. In this paper we reinvestigated the Hodgkin-Huxley model through computer simulation and determined the threshold potentials by applying different types of stimulating input signals. To implement the work, a computer programme of the Hodgkin-Huxley model was written in MATLAB programming language. The action potentials of neuron cells were checked and the threshold potentials of the neuron cell for specific types of stimulating input signals were tabulated with an aim to utilize these values to do experiment on neuron cell in future.
\end{abstract}

Keywords: Neuron, Membrane, Ionic Current, Kinetics

\section{Introduction}

Our present day understanding and methods of neural excitability have been significantly influenced by the landmark work of Hodgkin and Huxley ${ }^{1-4}$. The HodgkinHuxley $(\mathrm{HH})$ model has far reaching impact on many different life science sub-disciplines. These include not only neurophysiology but also endocrinology, muscle and cardiac physiology, and developmental biology. Hodgkin and Huxley formulated their famous model assuming that the membrane gets active and inactive in time depending on the voltage; and the ion permeation processes occurring within the membrane are approximately continuous and deterministic ${ }^{1,2}$. The ionic currents and electrical signals generated by neuronal membranes are very important in the nervous system. These currents and signals also play important roles in affecting cellular functions such as secretion, contraction, migration etc. The voltage-dependent conductance discovered by Hodgkin and Huxley and the ionic channels which they imagined are ubiquitous in the cells of animals and plants.

Due to its simplicity and experimentally testable capability Hodgkin-Huxley model $(\mathrm{HH})$ held resilient for half a century. However, the $\mathrm{HH}$ model has several weaknesses ${ }^{5-7}$. The most important is this model is limited to only two voltage-dependent currents found in the squid giant axon. However, new currents must be added if we want this model to deal with excitable soma and dendrites of neurons ${ }^{5}$. In addition, Hodgkin and Huxley did not capture the kinetics of $\mathrm{Na}^{+}$channel correctly ${ }^{7}$. The $\mathrm{Na}^{+}$permeation-process within the active membrane are known neither continuous nor deterministic ${ }^{5}$. The active membrane is studied with discrete ion channels undergoing random fluctuation between open and closed stable states ${ }^{8}$. Moreover, $\mathrm{HH}$ model cannot properly explore the collective phenomena in neuronal networks 5 .

However, the experimental and theoretical developments of the past 20 years force researchers to re-evaluate the usefulness of $\mathrm{HH}$ model. Several researchers have proposed various modifications of the $\mathrm{HH}$ model ${ }^{7,9}$ and some other researchers proposed alternative neuron models ${ }^{10,11}$. But there are still many unresolved questions specifically in the human neurobiology such as cell surface area, number of ionic channels etc. which were not addressed properly by the HH model.

In this paper we have reinvestigated the $\mathrm{HH}$ model and will present the outcome of our works.

\section{The Model}

The Hodgkin-Huxley $(\mathrm{HH})$ model is based on the idea that the electrical properties of a segment of nerve membrane can be modeled by an equivalent circuit (fig.1). In the equivalent circuit, the current-flow across the membrane has two major components, one associated with charging the membrane capacitance and other associated with the movement of specific types of ions across the membrane. The ionic current is further subdivided into three distinct components, a sodium current $I_{N a}$, a potassium current $I_{K}$, and a small leakage current $I_{L}$ that is primarily carried by chloride ions ${ }^{1}$

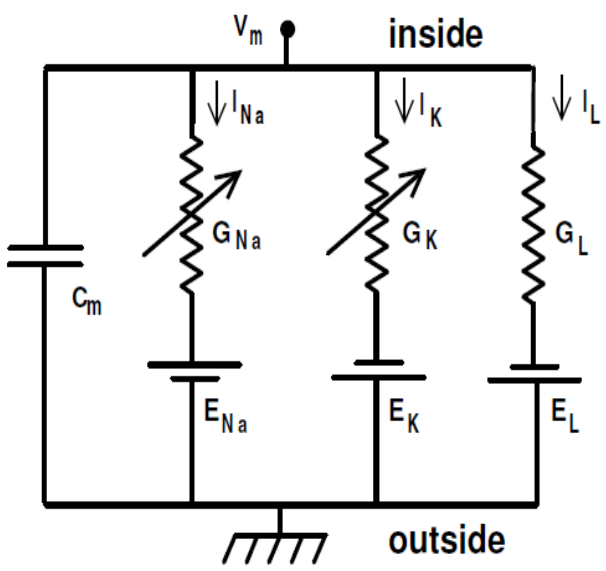

Fig. 1. Shows electrical equivalent circuit of a nerve membrane ${ }^{5}$.

The electrical properties of the nerve membrane shown in figure 1 are expressed mathematically ${ }^{5}$ by the differential equation (1).

$C_{m} \frac{d V_{m}}{d t}+I_{\text {ion }}=I_{\text {ext }}$

where, $\mathrm{C}_{\mathrm{m}}$ and $\mathrm{V}_{\mathrm{m}}$ represent the membrane (lipid bilayer) capacitance and the membrane potential respectively, $\mathrm{I}_{\mathrm{ion}}$ is

"Author for correspondence. e-mail: naimulphy@du.ac.bd 
the total ionic current and $\mathrm{I}_{\mathrm{ext}}$ is the external input or exciting current.

The total ionic current, $I_{\text {ion, }}$ in equation (1) is the algebraic sum of the individual contributions from all participating ion types (equation (2)):

$I_{\text {ion }}=\sum_{k} I_{k}=\sum_{k} G_{k}\left(V_{m}-E_{k}\right)$

In equation (2), $k$ represents the channel types and $G_{k}$ represents macroscopic conductance ${ }^{2}$ generated due to channels of type $\mathrm{k}$ and $\mathrm{E}_{\mathrm{k}}$ represents the reversal voltage sources whose voltages are determined by the ratio of the intra- and extra-cellular concentration of ionic species of interest transport through channels of type $\mathrm{k}$.

In the original work of Hodgkin-Huxley $(\mathrm{HH})$ model based on Squid giant axon, there are three such channels or currents $^{9}$ - sodium current $I_{N a}$, potassium current $I_{K}$, and a leakage current $I_{L}$ (equation (3)):

$I_{\text {ion }}=G_{N a}\left(V_{m}-E_{N a}\right)+G_{K}\left(V_{m}-E_{K}\right)+G_{L}\left(V_{m}-E_{L}\right)$

Each individual ion channel was thought of as containing a small number of physical gates that regulate the flow of ions through the channel. An individual gate can be in one of two states, permissive or non-permissive. When all of the gates for a particular channel are in the permissive state, ions can pass through the channel and the channel is open. If any of the gates are in the non-permissive state, ions cannot flow and the channel is closed $d^{9}$. If gates of a particular type $\boldsymbol{i}$ are considered, a probability $p_{i}$ can be defined ranging between 0 and 1 , which represents the probability of an individual gate being in the permissive state and $\left(1-p_{i}\right)$ as the fraction in the non-permissive state. Transitions between permissive and non-permissive states in the $\mathrm{HH}$ model were assumed to obey first-order kinetics ${ }^{10}$ and are expressed mathematically by the equation (4).

$\frac{d p_{i}}{d t}=\alpha_{i}(V)\left(1-p_{i}\right)-\beta_{i}(V) p_{i}$

where, $\alpha_{i}$ and $\beta_{i}$ are rate constants for the $\mathrm{i}$-th ion channel, which depend on voltage but not time.

The macroscopic conductance, $G_{k}$ due to channel of type $k$, with constituent gates of type $i$, is proportional to the product ${ }^{2}$ of the individual gate probabilities $p_{i}$ and is expressed by equation (5).

$G_{k}=\overline{g_{k}} \prod_{i} p_{i}$

where, $\overline{g_{k}}$ is a normalization constant that determines the maximum possible conductance when all the channels are in open state.

Hodgkin and Huxley modelled the sodium conductance using three gates ${ }^{5}$ of a type labeled ' $m$ ' and one gate of type ' $h$ ' and applying these to the sodium channels using both the generalized and the standard notation ${ }^{3}$ yields equation (6).

$G_{N a}=\overline{g_{N a}} p_{m}^{3} p_{h}=\overline{g_{N a}} m^{3} h$

Similarly, the potassium conductance is modelled with four identical ' $n$ ' gate is expressed by equation (7):

$G_{K}=\overline{g_{K}} p_{n}^{4}=\overline{g_{K}} n^{4}$
Using the value of $\mathrm{G}_{\mathrm{Na}}, \mathrm{G}_{\mathrm{K}}$, and $\mathrm{G}_{\mathrm{L}}$, the ionic current, $I_{\text {ion }}$ in $\mathrm{HH}$ model can be written in standard notation as (equation (8)):

$$
\begin{aligned}
& I_{\text {ion }}=\overline{g_{N a}} m^{3} h\left(V_{m}-E_{N a}\right)+\overline{g_{K}} n^{4}\left(V_{m}-E_{K}\right)+ \\
& \overline{g_{L}}\left(V_{m}-E_{L}\right)
\end{aligned}
$$

Now, using equation (8), the electrical properties in nerve membrane in equation (1) can be expressed as-

$$
\begin{aligned}
& \frac{d V}{d t}=-\frac{1}{C}\left[\overline{g_{N a}} m^{3} h\left(V_{m}-E_{N a}\right)+\overline{g_{K}} n^{4}\left(V_{m}-E_{K}\right)+\right. \\
& \left.\overline{g_{L}}\left(V_{m}-E_{L}\right)+I\right]
\end{aligned}
$$

Therefore, we can summarize the $\mathrm{HH}$ model by the following 4 non-linear ordinary differential equations (equation (10), (11), (12) and (13)):

$$
\begin{aligned}
& \frac{d V}{d t}=-\frac{1}{C}\left[\overline{g_{N a}} m^{3} h\left(V_{m}-E_{N a}\right)+\overline{g_{K}} n^{4}\left(V_{m}-E_{K}\right)+\right. \\
& \frac{d m}{d t}=\alpha_{m}(V)(1-m)-\beta_{m}(V) m \\
& \frac{d h}{d t}=\alpha_{h}(V)(1-h)-\beta_{h}(V) h \\
& \frac{d n}{d t}=\alpha_{n}(V)(1-n)-\beta_{n}(V) n
\end{aligned}
$$

The equation (10) represents the change of membrane potential with time and the equation (11), (12), and (13) represent the transition probabilities of ' $m$ ', ' $h$ ' and ' $n$ ' type of gates of sodium ( $\mathrm{m}$ and $\mathrm{h}$ ) and potassium ( $\mathrm{n}$ ) ions respectively between the permissive and non-permissive states.

\section{Materials and Methods}

The equivalent circuit (fig. 1) of the Hodgkin-Huxley (HH) model was mathematically represented by four coupled ordinary differential equations (equation (10), (11), (12), and (13)). In our work, we solved the ordinary differential equations of the HH model using MATLAB and presented the membrane potential of axon and the gating variables with respect to time. To solve the Hodgkin and Huxley equations the MATLAB function 'ode45' which is MATLAB's standard solver for ordinary differential equation (ODEs), was used. The built-in function 'ode45'implements a Runge-Kutta method with a variable time step to optimize the computation in order to achieve an efficient result. For our work, we wrote sets of different MATLAB codes to stimulate the neuron by input signals, then to solve the coupled differential equations of $\mathrm{HH}$ model by calling the solver function 'ode45' and to plot the results.

We applied two types input signal to excite the neuron cell.

First, we applied 'constant bias' voltage signal and after that 'sinusoidal bias' voltage signal was applied. For the constant bias voltage signal the amplitude and polarity of the input signal was varied and the firing of neuron cell was observed. For the sinusoidal bias voltage signals both the peak amplitude and frequency of the input signal were varied and the firing response of neuron was observed. If the neuron was fired, then from observing the action potential we tabulated the threshold values of voltage and frequency at which the neuron was fired. 
For our works the simulation parameters were chosen to be identical to those values for squid axonal membrane used by Hodgkin and Huxley in their seminal paper ${ }^{2}$ (table 1).

Table 1. Shows the parameters chosen for simulation

\begin{tabular}{lll}
\hline $\mathrm{gNa}$ & Maximum sodium conductance & $120 \mathrm{mScm}^{-2}$ \\
$\mathrm{gK}$ & Maximum potassium conductance & $36 \mathrm{mScm}^{-2}$ \\
$\mathrm{gl}$ & Leakage conductance & $0.3 \mathrm{mScm}$ \\
$\mathrm{ENa}$ & Sodium Nernst potential & $55 \mathrm{mV}$ \\
$\mathrm{EK}$ & Potassium Nernst potential & $-72 \mathrm{mV}$ \\
$\mathrm{E}_{\mathrm{L}}$ & Leakage Nernst potential & $-49.387 \mathrm{mV}$ \\
$\mathrm{C}_{\mathrm{m}}$ & Membrane capacitance & $1 \mu \mathrm{Fcm}^{-2}$
\end{tabular}

\section{Analysis and Results}

Results of our work for measuring the threshold potentials using constant bias voltage and sinusoidal input voltage signals were presented separately. First we presented the results for constant bias voltage, after that we presented the results of sinusoidal input voltage signals. For the sinusoidal voltage signal first we considered the results for input signal of 'constant frequency with variable voltage amplitude' and then the results of 'variable frequency with constant voltage amplitude' were considered.

All the figures depicted here were drawn in MATLAB and in every figure, the top one represented action potentials in $\mathrm{mV}$ with the change of time measured in $\mathrm{mS}$, the middle one represented the change of normalized gate probabilities $\mathrm{m}$, $\mathrm{n}$, and $\mathrm{h}$ with time and the bottom one represented the stimulating input signal in $\mathrm{mV}$.

\section{IV (A). Analysis of Results with Constant Voltage Input Signals}

We started our simulation with injecting a current from -10 $\mathrm{mV}$ (fig. 2). The fig. 2 shows that potassium gating probability(n) increases just after a few milliseconds and it reaches up to 0.772 . This caused the $\mathrm{K}+$ ion gates to open. Fig. 2 also shows that $\mathrm{Na}+$ gate activation probability (m) shoots up to almost 1 and the $\mathrm{Na}+$ gate inactivation probability (h) decreases down to 0.078 . This caused the $\mathrm{Na}+$ ion gates to open. These resulted in a rapidly increasing spike in the membrane action potential shown in figure 2 (top one). The action potential in fig. 2 is greater than the injected input voltage, because the neuron created a voltage itself and added it to the injected voltage. It signifies that a large amount of information can pass through the neurons much faster when the neuron is fired. From fig. 2 we can also notice that the change in membrane potential is almost similar in shape to the change in gating activation probability of sodium (Na+). After firing once, sodium gating probability $(\mathrm{m})$ increased slowly and after $15.003 \mathrm{mS}$, the $\mathrm{Na}+$ gate activation probability increased rapidly again and caused the neuron to fire again. However, the maximum value of membrane action potential reached slightly less than the maximum value of the previous firing. The neuron fired almost after every $15 \mathrm{mS}$ and the maximum peak value of the action potential was almost $50 \mathrm{mV}$.

Therefore, if constant $-10 \mathrm{mV}$ input signal was injected, the neuron fired continuously and allowed to pass a huge amount of information in a very short time interval.

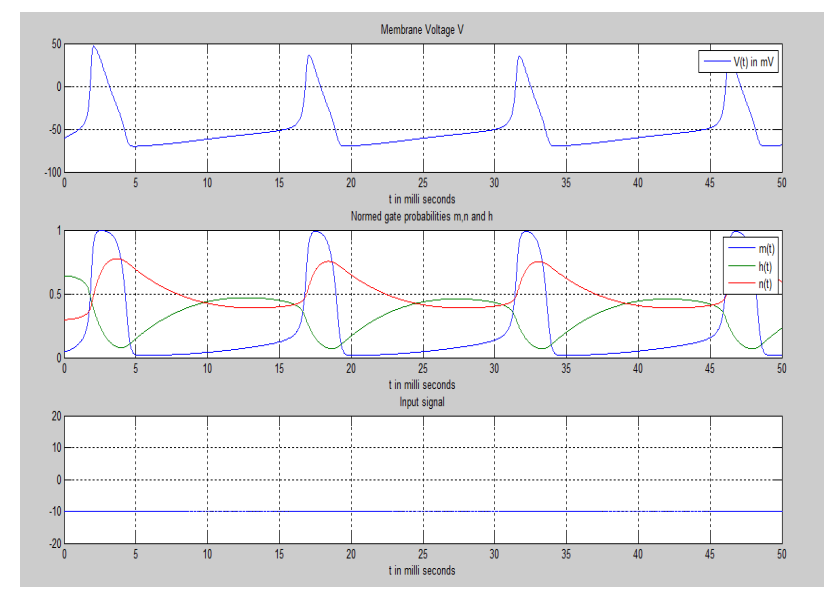

Fig. 2. Shows the membrane potential, the gate probabilities, and the constant bias input signal of $-10 \mathrm{mV}$.

For injecting a voltage of $-2 \mathrm{mV}$ (fig. 3) the neuron fired only once. The maximum value of the action potential for the input signal of $-2 \mathrm{mV}$ was $44.26 \mathrm{mV}$.

For injecting a signal of voltage $-1 \mathrm{mV}$ (fig. 4), the neuron did not fire at all. The graph of membrane potential levelled out at $-59.19 \mathrm{mV}$.

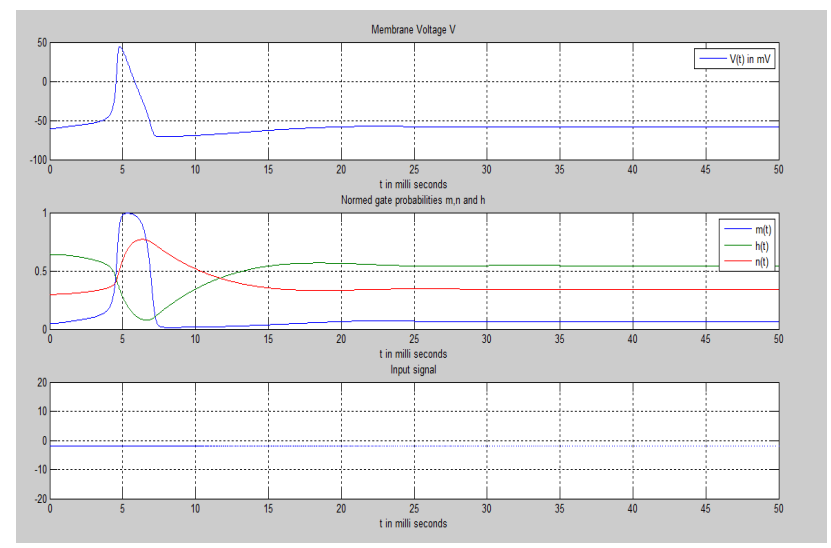

Fig. 3. Shows the membrane potential, gate probabilities, and the constant amplitude input signal of $-2 \mathrm{mV}$.

To check whether the neuron fired or not for other constant input voltage signals, we injected voltage signals of $3 \mathrm{mV}, 5$ $\mathrm{mV}$ and $10 \mathrm{mV}$. But it never fired.

Therefore, the threshold potential for constant bias input signal was $-2 \mathrm{mV}$. 


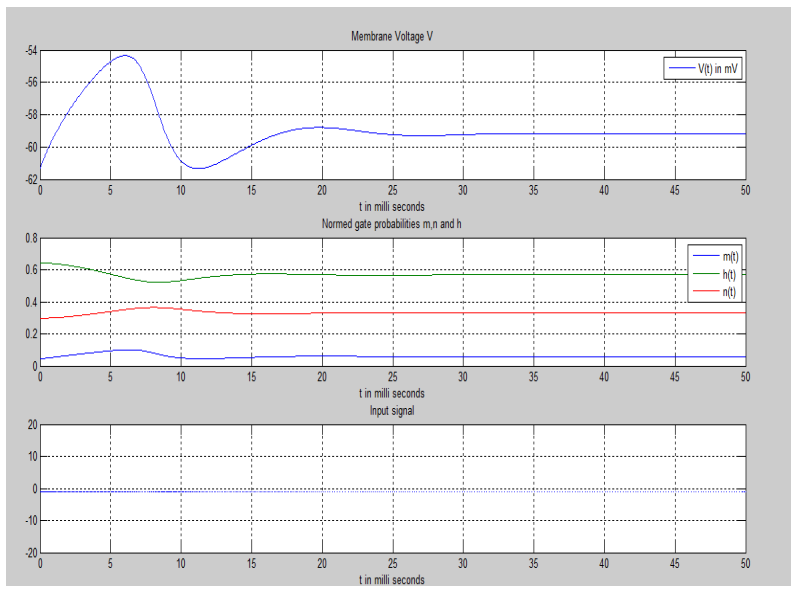

Fig. 4. Shows the membrane potential, gate probabilities, and the constant amplitude input signal of $-1 \mathrm{mV}$.

\section{IV (B). Analysis of Results with Sinusoidal Input Voltage Signals:}

Results for the constant frequency with variable voltageamplitude input signals:

To inject a sinusoidal voltage, we first fixed the frequency at $1 \mathrm{~Hz}$ and then changed the voltage amplitude to various values. We started injecting sinusoidal voltage from $1 \mathrm{mV}$, but until injecting the voltage of $2.5 \mathrm{mV}$ the neuron did not fire. From fig. 5 we can see for sinusoidal input with $2.5 \mathrm{mV}$ peak amplitude and $1 \mathrm{~Hz}$ frequency, the neuron fired for once with a rapid rise of potential followed by a rapid fall and giving a maximum action potential of $39.9 \mathrm{mV}$. Fig. 5 also shows that once the neuron fired, the gate activation probability of the sodium and potassium ions ( $m$ and $n$ ) increased and inactivation probability (h) of the sodium ions decreased. After firing only once the neuron did not fire at all and the action potential and activation and inactivation probabilities settled down to sinusoidal waves with slightly different amplitudes.

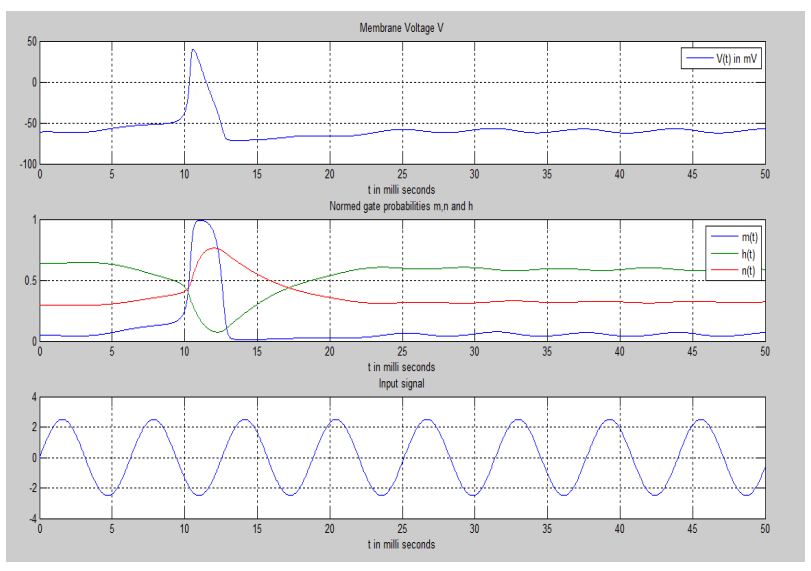

Fig. 5. Shows the membrane potential along with the gate probabilities and the injected sinusoidal input signal with $1 \mathrm{~Hz}$ frequency and with $2.5 \mathrm{mV}$ peak-amplitude.

We kept increasing the peak-amplitude of the applied sinusoidal signal. At peak-amplitude of $4.2 \mathrm{mV}$, the neuron fired for twice. The maximum value of the action potential was $45.84 \mathrm{mV}$ with $20.933 \mathrm{mS}$ time duration between two consecutive firing.

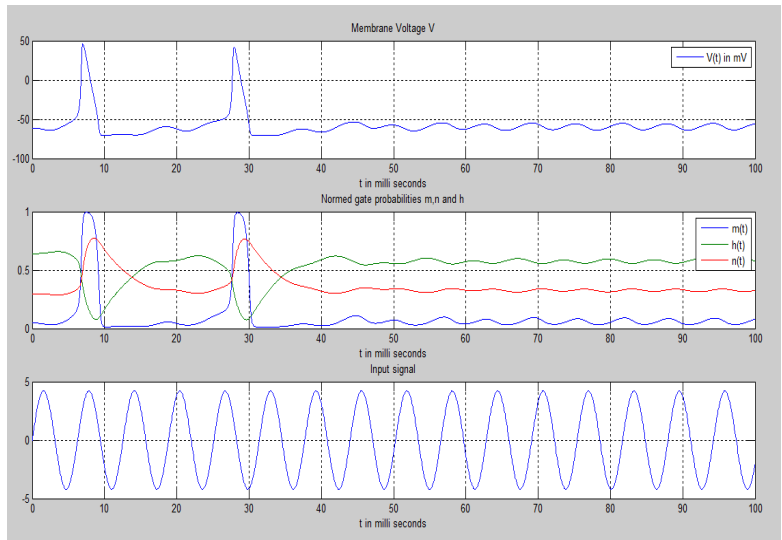

Fig. 6. Shows the membrane potential along with gate probabilities and the injected sinusoidal input signal with $1 \mathrm{~Hz}$ frequency and with $4.2 \mathrm{mV}$ peak-amplitude.

As our aim was to find an input signal capable of providing a continuous firing, we kept increasing the peak-amplitude of applied sinusoidal input signal.

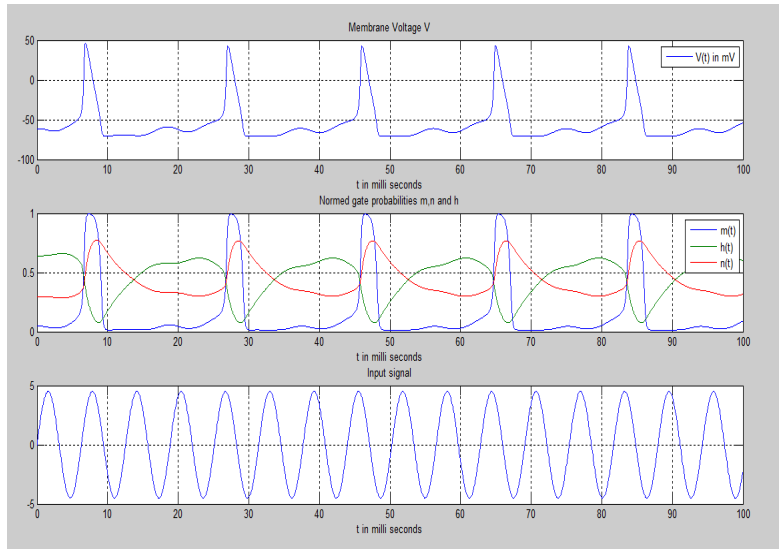

Fig. 7. Shows the membrane potential along with the gate probabilities and the injected sinusoidal input signal with $1 \mathrm{~Hz}$ frequency and with $4.5 \mathrm{mV}$ peak-amplitude.

At $4.5 \mathrm{mV}$ peak-amplitude (fig. 7) the neuron fired continuously and provided maximum action potential of $46.07 \mathrm{mV}$.

Therefore, the threshold potential for sinusoidal input signals with constant $1 \mathrm{~Hz}$ frequency was $2.5 \mathrm{mV}$.

Results for the constant voltage peak-amplitude with variable frequency sinusoidal input signals:

To examine the effect of different frequencies on neuron firing, we first fixed the amplitude at $1 \mathrm{mV}$ and then started changing the frequency from $1 \mathrm{~Hz}$. But the neuron never fired at all. Then we fixed the peak-amplitude at $5 \mathrm{mV}$ and changed the frequency but it has behaved the same way as before. So, we kept increasing the peak-amplitude and changed the frequency. If we fixed the peak-amplitude at 10 $\mathrm{mV}$ and kept changing the frequency, we found that at 1.45 $\mathrm{Hz}$ the neuron fired continuously with a small decrease in amplitude (fig. 8). 


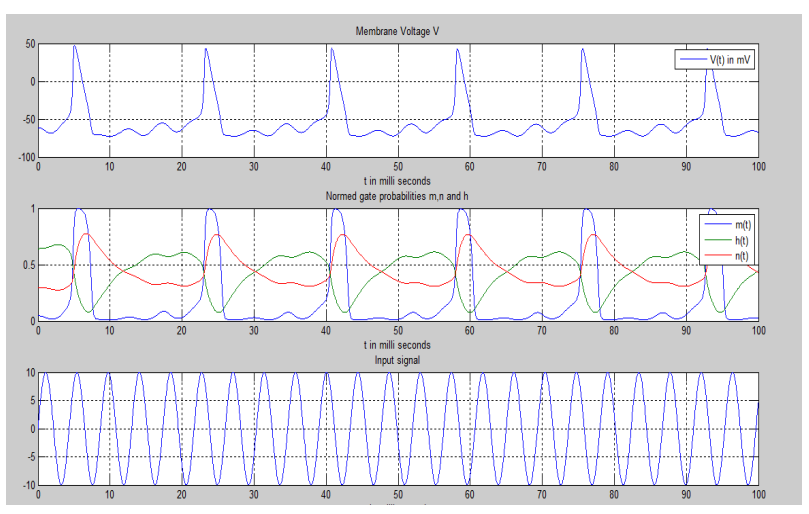

Fig. 8. Shows the membrane potential along with the gate probabilities and injected sinusoidal input signal with $10 \mathrm{mV}$ peakamplitude and with $1.45 \mathrm{~Hz}$ frequency.

The obtained maximum value of the firing potential was $47.18 \mathrm{mV}$ and the duration between two consecutive firing was $18.218 \mathrm{mS}$ (fig. 8). As we kept increasing the frequency, the number of firing decreased. At frequency $1.52 \mathrm{~Hz}$ (fig. 9), the neuron fired only for once and the obtained maximum action potential was $46.93 \mathrm{mV}$.

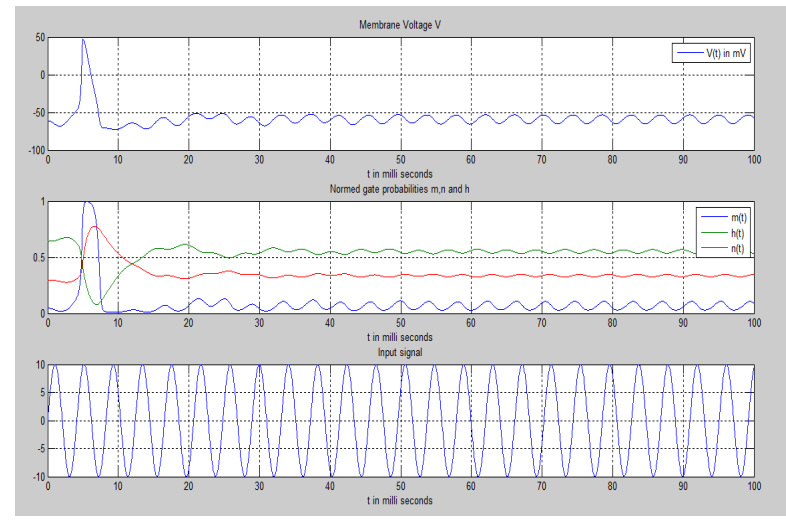

Fig. 9. Shows the membrane potential along with the gate probabilities and the injected sinusoidal input signal with $10 \mathrm{mV}$ peak-amplitude and with $1.52 \mathrm{~Hz}$ frequency.

We kept increasing the input frequency and found that up to $2.34 \mathrm{~Hz}$, neuron fired once each time, however, at applied frequency $2.35 \mathrm{~Hz}$ (fig. 10), the neuron stopped firing.

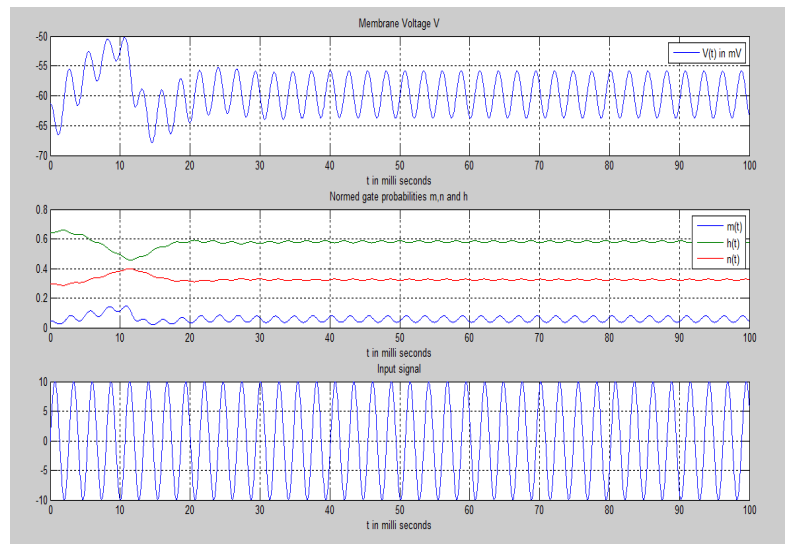

Fig. 10. Shows the membrane potential along with the gate probabilities and the injected sinusoidal input signal with $10 \mathrm{mV}$ peak-amplitude and with $2.35 \mathrm{~Hz}$ frequency.
Therefore, for sinusoidal input signal with constant $10 \mathrm{mV}$ peak-amplitude, the frequency threshold was $2.34 \mathrm{H}_{3}$.

It should be noted that the gating probabilities for the sinusoidal bias input signal behaved very much similar to those for the constant bias input voltage signal, where the activation probabilities peaked at maximum and the inactivation probabilities down to minimum when the neuron fired. Biologically this corresponds to the opening and shutting down of the ion gates so that sodium and potassium ions can flow freely through the neuron, passing the information much more efficiently.

\section{Discussion}

Our research involved using Hodgkin and Huxley's model to find the threshold membrane potential. A periodic firing of the cell was observed if the applied 'constant bias' input stimulating voltage signal had high enough amplitude (i.e. $2 \mathrm{mV}$ ) or if the input 'sinusoidal stimulating signal' has high enough voltage amplitude (i. e. amplitude $2.5 \mathrm{mV}$ ) and low enough frequency (i. e. frequency $1 \mathrm{~Hz}$ ). The important property of the action potential "all or nothing" event is verified in our simulation using $\mathrm{HH}$ model which tells that the cell is triggered only above the certain threshold voltage. Further, there is a minimal recovery time of the cell until another action potential can be prompted.

It should also be noted that with the gradual increase of frequency from $1.45 \mathrm{~Hz}$ (keeping the voltage amplitude fixed at $10 \mathrm{mV}$ ), the obtained number of firing decreased. For the applied signal of frequency $1.50 \mathrm{~Hz}$, we obtained two firings. But at frequency $1.51 \mathrm{~Hz}$, suddenly the number of firings increased and we obtained three firings. Then again at frequency $1.52 \mathrm{~Hz}$, we obtained only one firing. This phenomenon proves the bifurcation ${ }^{14,15}$ nature of the $\mathrm{HH}$ model which means that either the neuron is not firing at all or firing at a minimum rate. Because the "all or nothing" principle says there is no smooth increase in action potential but there is a sudden jump in amplitude.

The simulation results for the action potentials of our present work match the current knowledge of action potential. Our simulation solutions could be used to model the propagation of action potential when there is a change in certain parameter or in intensity of an input wave.

\section{Conclusion}

In this paper we have used computer simulation and analysis to know the theoretical mechanism that exists in neuron cells. For our simulation works we applied two types of stimulations: the constant bias voltage and the sinusoidal voltage. However, to know the exact behavior of the neuron we should also apply more types of stimulating input signals to stimulate the neuron cell such as single pulse stimulation, double pulse stimulation, and exponential stimulation with variable time constant. Then, we will know the threshold voltages of the neuron for that specific input signal and can choose the optimum stimulating input signal for a specific neuron. In future the standard $\mathrm{HH}$ model based on only $\mathrm{Na}+$ and $\mathrm{K}+$ ion channels should also be compared through computer simulations to the alternative model based on the 
ion channel populations represented through Markov process.

\section{References}

1. Hodgkin A L, A F., Huxley 1952. Currents carried by sodium and potassium ions through the membrane of the giant axon of Loligo. J Physiol, 116 (4), 449-472.

2. Hodgkin A L, A F., Huxley 1952. The components of membrane conductance in the giant axon of Loligo. J Physiol, 116 (4), 473-496.

3. Hodgkin A L, A F., Huxley 1952. The dual effect of membrane potential on sodium conductance in the giant axon of Loligo. $J$ Physiol, 116 (4), 497-506.

4. Hodgkin A L, A F, Huxley 1952. Katz B. Measurement of current-voltage relations in the membrane of the giant axon of Loligo. J Physiol, 116 (4), 424-448.

5. Meunier C and I., Segev 2002. Playing the Devil's advocate: is the Hodgkin-Huxley model useful? TRENDS in Neurosciences, 25(11), 558-563.

6. Naundorf B, F, Wolf and M. Volgushev 2007. Hodgkin and Huxley model - still standing? Nature, 445, E1-E2.

7. Strassberg A F and L J. De Felice 1993. Limitations of the Hodgkin-Huxley formalism: effects of single channel kinetics on transmembrane voltage dynamics. Neural computation, 5(6), 843-855.

8. Hille B., 1992. Ionic Channels of Excitable Membrane, 2nd ed. Sinauer Associates, Sunderland, MA.
9. Kistler M W, W, Gerstner von Hemmen L J., 1997. Reduction of the Hodgkin-Huxley equations to a single-variable threshold model. Journal Neural Computation Archive, 9(5), 1015-1045.

10. Heimburg T and A D. Jackson 2005. On soliton propagation in biomembranes and nerves. Proc Natl AcadSci USA, 102(28), 9790-9795.

11. Appali R, van U, Rienen T. A., Heimburg 2012. Comparison of the Hodgkin-Huxley Model and the Soliton Theory for the Action Potential in Nerves. In: Advances in Planar Lipid Bilayers and Liposomes, 16, 275-299.

12. Mondeel T., 2012. Modelling Neuronal Excitation: The Hodgkin-Huxley Model. (Thesis work, University of Amsterdam)

13. Nelson M E., 2004. Electrophysiological Models In: Databasing the Brain: From Data to Knowledge. (S. Koslow and S. Subramaniam, eds.) Wiley, New York.

14. FeudelU,Neiman A, X, Pei W, Wojtenek H, Braun M, Huber F. Moss 2000. Homoclinic bifurcation in a Hodgkin-Huxley model of thermally sensitive neurons. Chaos, 10(1), 231-239.

15. Hassard B., 1978. Bifurcation of Periodic Solutions of the Hodgkin-Huxley Model for the Squid Giant. Axon J. theor. Biol, 71, 401-420. 